Los efectos de la crisis sobre el sistema escolar

The effects of economic crisis on the school system 


\title{
Introducción al debate "Los efectos de la crisis sobre el sistema escolar" I
}

\section{Introduction to the controversy 'The effects of economic crisis on the school system'}

\author{
Julio Carabaña \\ Universidad Complutense de Madrid. España/Spain \\ carabanya@ccedu.ucm.es
}

La primera idea sobre la relación entre crisis económicas y matrícula escolar que nunca tuve en la cabeza procede (si no recuerdo mal, claro está) de The Economics of Education, de John Vayzey, publicado en 1962 en Londres por Faber and Faber. Allí se hace notar la escasa elasticidad a la baja de la matrícula universitaria, que en Estados Unidos apenas habría descendido un tres por ciento durante la Gran Depresión de 1929, muy poco comparado con la elasticidad al alza. Aún así, ambas elasticidades son positivas, de modo que los estudios universitarios se comportaban, en aquellos tiempos y en Estados Unidos, como un bien normal, cuyo consumo aumenta y disminuye con la renta, si bien menos que proporcionalmente.

En las recesiones posteriores parece que la pauta ha ido cambiando y que la escolarización se ha ido volviendo contracíclica. Así, en un estudio reciente sobre la crisis en Estados Unidos, se da por consabido que la matrícula en los colleges tiende a aumentar en las recesiones (Long, 2012). Quizás los estudios más favorables a esta generalización sean los de Dellas y Sakelaris (2003) y Dellas y Koubi (2003), que estudian en Estados Unidos el ingreso en college en el periodo 1968-1988, durante el cual acontecieron cuatro recesiones; su resultado más fuerte es que en la serie temporal con datos agregados un uno por ciento de variación en el desempleo total da lugar a un 0,57 \% de cambio en la matrícula; concluyen que los cambios en el coste de oportunidad asociados al ciclo desempeñan un papel principal en la escolarización. Pero otros estudios son menos concluyentes. Johnson (2013), también para Estados Unidos, encuentra una pauta contracíclica en los hombres, pero acíclica entre las mujeres. En la 'Gran Recesión' de 2008, Long (2012), aprovechando las variaciones por Estados, encuentra que los alumnos de college a tiempo completo han disminuido, debiéndose el aumento general a los estudiantes a tiempo parcial, que ha ocurrido pese al incremento del coste de la matrícula, no compensado por el de las becas (Long, 2012). En Gran Bretaña, Clark (2011) explica por la bonanza económica de los noventa que se desacelerara el crecimiento de matrícula universitaria, atreviéndose a predecir que un empeoramiento de las condiciones de trabajo lo aceleraría. Una síntesis prudente de los estudios empíricos podría ser que la relación se detecta, pero no siempre ni con mucha intensidad.

¿Cómo se explica que los estudios superiores hayan pasado a convertirse en el análogo a un 'bien inferior', cuyo consumo crece cuando los ingresos bajan? El planteamiento más senciIlo es que su demanda depende de dos fuerzas opuestas. Por un lado está la renta doméstica y los costes directos; por otro lado están los costes de oportunidad, cuyo principal componente son los ingresos del trabajo a que renuncian los estudiantes. En las recesiones, bajan los costes de oportunidad, pero también disminuye la renta de los hogares y su capacidad para hacer frente a los costes directos de estudiar. En los períodos de auge ocurre a la inversa: las familias tienen más fácil afrontar los costes de los estudios, pero si lo hacen tienen que renunciar a mayores in- 
gresos del mercado. La matrícula variará con el ciclo económico si domina el efecto de la renta y los costes directos, y contra el ciclo si domina el efecto del coste de oportunidad. El modelo puede sintetizarse en una ecuación como la siguiente:

$P(E)=a+b 1 a n ̃ o+b 1$ renta doméstica $+b 2$ costes directos+b3 coste de oportunidad (1)

donde se espera que los coeficientes de los costes sean negativos y el de la renta doméstica positivo; en todo caso, si la escolarización solo dependiera de estos factores, el coeficiente de los años sería nulo. El efecto que domine dependerá tanto del tamaño de los coeficientes como de la magnitud de los cambios en las variables. A partir de aquí, se abren las interrogaciones. ¿Por qué no varían a la par los ingresos de los hogares y los ingresos de los jóvenes? ¿Por qué varían en el espacio y en el tiempo la importancia relativa del coste de oportunidad y la renta doméstica?

En España, la escolarización parece haber sido indiferente al ciclo económico desde fines del siglo xx hasta 2009, años en los que se estancó; luego ha sido contracíclica, pues aumentó fuertemente en los años de la crisis, especialmente en los primeros, y parece que ha vuelto a estancarse en los años posteriores a 2013, en los que se ha iniciado la recuperación. La Tabla 1 presenta datos básicos que acreditan esta relación. Muestra que las tasas netas de escolarización de dos grupos de edad, 15-19 y 18-24 años aumentaron 11,2 puntos y 16,3 puntos respectivamente justo entre 2008 y 2014, pero no antes y apenas después. Estas tasas netas de escolarización son mejor indicador que el abandono escolar temprano, que sin embargo es el más conocido y empleado. La Tabla 1 muestra que ha evolucionado de modo análogo hasta 2014, pero que continúa disminuyendo todavía en 2016; esta diferencia se explica porque el abandono temprano es un indicador mixto, compuesto de escolarización y titulación en la población de 18 a 24 años (Carabaña, 2016). La separación de las tasas netas en dos grupos de edad indica que la evolución ha sido la misma para todos los niveles de estudio, incluyendo los universitarios.

Tabla 1. Evolución de las tasas netas de escolaridad por grupos de edad y del abandono temprano

\begin{tabular}{lccc}
\hline AÑOS & $15-19$ años & $18-24$ años & Abandono \\
\hline 2004 & 76,6 & 40,8 & 32,2 \\
\hline 2005 & 74,3 & 39,7 & 31 \\
\hline 2006 & 75,1 & 40,2 & 30,3 \\
\hline 2007 & 74,9 & 39,9 & 30,8 \\
\hline 2008 & 76,1 & 40,2 & 31,7 \\
\hline 2009 & 78,4 & 42,2 & 30,9 \\
\hline 2010 & 81,3 & 45,9 & 28,2 \\
\hline 2011 & 83 & 48,7 & 26,3 \\
\hline 2012 & 85,1 & 51,8 & 24,7 \\
\hline 2013 & 85,8 & 53,6 & 23,6 \\
\hline 2014 & 87,3 & 56,5 & 21,9 \\
\hline 2015 & 87,2 & 56,7 & 20 \\
\hline 2016 & 87,9 & 57,7 & 19 \\
\hline VARIACIONES DE 2008 A 2014 & 11,2 & 16,3 & $-9,8$ \\
\hline & $0,1,2$ & $-2,9$ \\
\hline VARIACIONES DE 2013 A 2016 & 0,6 & \\
\hline
\end{tabular}

Fuente: Eurostat, edat_Ifse_19 y edat_Ifse_14 
¿Cómo se ha ido explicando en España esta evolución de la escolarización? Quizás pueda decirse que el argumento del mercado de trabajo se ha impuesto con la crisis tras haberse insinuado durante el auge. Lo primero que se detectó fue el estancamiento del abandono escolar temprano desde principios de siglo. Lacasa (2006) lo atribuyó a la LOGSE, por el momento en que los titulados en Bachillerato y FP dejaron de crecer, incluso teniendo en cuenta solo la población nacida en España (Lacasa, 2008). En la misma dirección de la LOGSE apuntaron algunos economistas (Felgueroso y otros, 2013) y sociólogos (Martínez García, 2009). Pero el mismo Lacasa menciona ya la explicación por el mercado de trabajo, que ha sido discutida en varios estudios académicos (Aparicio, 2012; Lacuesta et al., 2012; Martínez García, 2013). Todavía en 2014, el que esto escribe presentaba la situación como 'desconcertante', y se preguntaba cómo en un contexto tan favorable de disminución de población, aumento de los recursos públicos y privados, mejora de las condiciones socioculturales de los hogares e intervenciones políticas cada vez más profundas no había aumentado la escolarización (Carabaña, 2015). Pero ya para entonces, la hipótesis del coste de oportunidad se imponía a la vista del mucho más desconcertante aumento de la escolarización tras la crisis. "La explicación de los costes de oportunidad es casi la única que puede dar cuenta del rápido incremento de la escolarización en los últimos años" (Fernández y Martínez, 2015).

Además, durante los últimos años de la crisis se han producido cambios internos al sistema educativo que también pueden haber influido en la evolución de la escolaridad. Algunos positivamente, como la FP Básica y la ampliación de las vías de acceso a la Universidad; la mayor parte, como los recortes en el gasto público, el aumento de los 'precios públicos' en las universidades, el endurecimiento de las condiciones para la conservación de las becas o la reordenación de los ciclos universitarios según Bolonia, contrarrestando el efecto del mercado de trabajo.

La cuestión general propuesta a la discusión es a qué puede deberse que las tasas de escola- rización hayan crecido tanto en los primeros años de la crisis y hayan tendido a estancarse en los últimos años. Más en particular, nos preguntamos por el efecto de la disminución de la renta, el aumento de los costes directos y la disminución de los costes de oportunidad en la evolución de la escolarización, particularmente en la universitaria. Esto abre la puerta a muchas otras cuestiones. ¿Cómo definen los jóvenes y sus familias los cambios de situación? ¿Difiere, y cómo, la respuesta a estos cambios por sexo, clase social o ingresos? ¿Cuál es la importancia de los factores económicos y cuál la de las políticas educativas? ¿0curre en otros países lo mismo que en España? Si no, ¿por qué? Cada uno de los debatientes ha abordado con preferencia alguna de estas cuestiones, intercambiando sus puntos de vista con los del resto en el curso de la redacción. Muchas han quedado sin tratar. El lector queda invitado a juzgar los resultados y a sumarse a la discusión.

\section{REFERENCIAS BIBLIOGRÁFICAS}

Aparicio, A. (2012). High-School Dropouts and Transitory Labor Market Shocks: The Case of the Spanish Housing Boom. IZA DP, N. ${ }^{0} 5139$.

Brown J. N., Hoxby C. M. (ed.) (2012) How the Financial Crisis and Great Recession Affected Higher Education. Chicago: University of Chicago Press.

Carabaña, J. (2015). El sistema de enseñanza. En Torres, C. (Coord.). La Sociedad española. CIS: Madrid.

Carabaña, J. (2016) ¿Aumentar la escolaridad 0 fomentar la titulación en Secundaria Superior? En FESE, Indicadores comentados sobre el estado del sistema educativo español, 2016 (pp. 45-51). Madrid: Fundación Ramón ArecesFundación Europea Sociedad y Educación.

Clark, D. (2011). Do Recessions Keep Students in School? The Impact of Youth Unemployment on Enrollment in Post-compulsory Education in England. Economica, 78 (311), 523-545.

Dellas, H., and Koubi, V. (2003). Bussines cycles and schooling. European Journal of Political Economy, 19 (4), 843-859. 
Dellas, H. and Sakellaris, P. (2003 ). On the Ciclycality of Schooling. Theory and Evidence. Oxford Economic Papers, 55 (1), 148-172.

Felgueroso, F., Gutiérrez, M., Jiménez, S. (2013). ¿Por qué el abandono escolar se ha mantenido tan elevado en España en las últimas dos décadas? El papel de la Ley de Educación (LOGSE). FEDEA Colección Estudios Económicos 2002-2013.

Fernández Mellizo-Soto, M., Martínez García, J. (2015). La evolución de la escolaridad y los títulos. En Torres, C. (ed). La sociedad española 2015. Madrid: CIS.

Johnson, M. T. (2013). The impact of business cycle fluctuations on graduate school enrollment. Economics of Education Review, 34, 122-134.

Lacasa, J. M. ( 2006) España es el tercer país de la UE que se queda fuera de los objetivos de Lisboa 2010. En http://www.magisnet.com/pdfs/ indicadores06.pdf.
Lacasa, J. M. (2008) El nivel de formación de los jóvenes cae al $61,1 \%$, el más bajo desde el año 1995. Magisterio Español, 14-6-2008.

Lacuesta, A., Puente, S., Villanueva, E. (2012). The Schooling Response to a Sustained Increase in LowSkill wages: Evidence from Spain 1989-2009. Documentos de Trabajo, N. ${ }^{0} 1208$, Banco de España.

Long, B. T. (2012). The Financial Crisis and College Enrollment: How Have Students and Their Families Responded? En Brown, J. R., Hoxby, C. M. (editors). How the Financial Crisis and Great Recession Affected Higher Education (pp. 209-233). Chicago: University of Chicago Press.

Martínez García, J. S. (2009). Fracaso Escolar, PISA y la Difícil ESO. RASE 2, 1, 56-85.

Martínez García, J. S. (2013). Estructura social y desigualdad en España. Madrid: La Catarata.

Vayzey, John (1962). The Economics of Education. London: Faber and Faber. 